“C 2019 IEEE. Personal use of this material is permitted. Permission from IEEE must be obtained for all other uses, in any current or future media, including reprinting/republishing this material for advertising or promotional purposes, creating new collective works, for resale or redistribution to servers or lists, or reuse of any copyrighted component of this work in other works." 


\title{
Energy Management and Time Scheduling for Heterogeneous IoT Wireless-Powered Backscatter Networks
}

\author{
Ngoc-Tan Nguyen ${ }^{12}$, Nguyen Van Huynh ${ }^{1}$, Dinh Thai Hoang ${ }^{1}$, Diep N. Nguyen ${ }^{1}$, Nam-Hoang Nguyen ${ }^{2}$, \\ Quoc-Tuan Nguyen ${ }^{2}$, and Eryk Dutkiewicz ${ }^{1}$ \\ ${ }^{1}$ School of Electrical and Data Engineering, University of Technology Sydney, Australia \\ 2 Vietnam National University, Hanoi, Vietnam
}

\begin{abstract}
In this paper, we propose a novel approach to jointly address energy management and network throughput maximization problems for heterogeneous IoT low-power wireless communication networks. In particular, we consider a low-power communication network in which the IoT devices can harvest energy from a dedicated RF energy source to support their transmissions or backscatter the signals of the RF energy source to transmit information to the gateway. Different IoT devices may have dissimilar hardware configuration, and thus they may have various communications types and energy requirements. In addition, the RF energy source may have a limited energy supply source which needs to be minimized. Thus, to maximize the network throughput, we need to jointly optimize energy usage and operation time for the Io $T$ devices under different energy demands and communication constraints. However, this optimization problem is non-convex due to the strong relation between energy supplied by the RF energy source and the IoT communication time, and thus obtaining the optimal solution is intractable. To address this problem, we study the relation between energy supply and communication time, and then transform the non-convex optimization problem to an equivalent convex-optimization problem which can achieve the optimal solution. Through simulation results, we show that our solution can achieve greater network throughputs (up to five times) than those of other conventional methods, e.g., TDMA. In addition, the simulation results also reveal some important information in controlling energy supply and managing low-power IoT devices in heterogeneous wireless communication networks.
\end{abstract}

Index Terms-Bistatic backscatter, RF energy harvesting, heterogeneous IoT, low-power communications, convex optimization.

\section{INTRODUCTION}

The Internet-of-Things (IoT) is a network of smart devices connected to the Internet to create favorable conditions for people's lives. Over the last few years, we have been experiencing an unprecedented development of IoT applications in all areas of life such as smart house, agriculture, industry 4.0, healthcare, and automotive. Cisco predicted that the number of IoT devices will exceed 50 billion by 2020 [1]. However, one of the biggest challenges for the development of IoT is energy management problem. Typically, IoT devices are powered by batteries and they use energy from the batteries for their communications. However, due to a massive number of IoT devices, replacing the batteries is very costly and sometimes impractical. Recently, wireless-powered backscatter networks (WPBNs) have been emerging as a prominent solution to tackle this problem [2]. In a WPBN, a wireless-powered device (WPD) can either harvest energy from the signals from an RF energy source to support its internal operations and transmissions, or backscatter the signals to transmit its information to the gateway. Due to a very small environmental footprint, wireless energy harvesting and backscatter communications are especially suitable to implement on low-power IoT communication networks. However, how to maximize the network performance in terms of the network throughput and energy consumption is an emerging challenge for the WPBNs.

To address this issue, the authors in [3] propose an algorithm to increase the bitrate of the backscatter devices. Intuitively, the backscatter transmitter adopts OOK modulation with FM0 encoding to transmit data. At the backscatter receiver, an algorithm using $\mathrm{CFO}$ correction technique and two components, i.e., a downsampling and a matched filter, is implemented to extract useful information from the received signals. The experimental results show that the proposed solution can achieve a bitrate of $2.5 \mathrm{kbps}$. Unlike [3], the authors in [4] use a frequency-shifted backscatter technique to reduce selfinterference at the backscatter receiver. In particular, this technique allows backscatter transmitter to shift to an adjacent frequency before reflecting signals. The experimental results demonstrate that the bitrate of backscatter devices can be enhanced up to $50 \mathrm{kbps}$. In [5], the authors introduce a multiantenna backscatter transmitter to eliminate the interference of ambient RF signals. At the backscatter receiver, a decoding algorithm is implemented to derive data from signals transmitted by multiple antennas. The key idea of the decoding algorithm is comparing the relative channel changes among the antennas, and thus the bits transmitted by the backscatter transmitter are derived. The experimental results show that the proposed backscatter design can achieve the bitrate up to $1 \mathrm{Mbps}$ at distances from 4 feet to 7 feet.

To address the energy management for WPBNs with multiple IoT devices, i.e., WPDs, multiple access mechanisms such as frequency-division multiplexing (FDM) and time-division multiplexing (TDM) are introduced to reduce the interference among WPDs [6], [7]. The experimental results show that the FDM and TDM techniques can reduce significantly the interference among WPDs, thereby increasing throughput for the whole network. Yet these solutions still only consider backscatter devices, and thus energy harvesting management for active transmissions is totally ignored. However, energy harvesting is a crucial part in low-power communication net- 
works that needs to be optimized. In [8], the authors propose a novel harvest-then-transmit (HTT) protocol to maximize the throughput of IoT devices through balancing energy harvesting and communication time. Nevertheless, backscatter communication devices are not considered in this work. Some recent research studies [9] and [10] have tried to address this problem by jointly optimizing backscattering, energy harvesting, and data transmission time. However, they only consider hybrid devices, i.e., the IoT devices are able to not only backscatter RF signals to transmit data, but also harvest energy for active transmissions. In practical, IoT devices are diverse with different hardware configuration and energy requirements that must be taken into account. Furthermore, in all aforementioned work, energy management is only optimized at IoT devices without considering impacts of the RF energy supply sources. However, this is also an important factor because transmission power of RF energy sources has major impact to the network performance of WPBNs (e.g., amount of energy harvested).

In this paper, we propose a new approach to address all aforementioned problems. In particular, we consider a heterogeneous IoT low-power wireless communication network in which different IoT devices have dissimilar hardware configuration with diverse energy requirements and communication constraints. The IoT devices can harvest energy from a dedicated RF energy source to support their transmissions or leverage the signals of the RF energy source to backscatter information to the gateway. We then formulate an optimization problem by jointly optimizing the time scheduling for the backscatter and HTT protocols together with the transmission power of the RF energy source. We show that the optimization problem is non-convex. By analyzing the characteristics of the network, we then can transform the non-convex optimization problem to an equivalent convex optimization problem, and thus the optimal solution for the whole network can be obtained. Through simulation results, we show that our solution can achieve greater network throughputs (up to five times) compared to other conventional methods (e.g., HTT protocols). In addition, the simulation results also reveal some important information in controlling energy and manging lowpower IoT devices in heterogeneous wireless communication networks. To the best of our knowledge, this is the first work in the literature which jointly addresses energy management and network throughput for heterogeneous IoT WPBNs.

\section{System MODEL}

In this paper, we consider a Heterogeneous WirelessPowered Backscatter Network (HWPBN) consisting of a dedicated RF energy source, multiple IoT devices (belonging to different types), and an IoT gateway that collects data from all IoT devices, as illustrated in Fig. 1. We consider three typical sets of IoT devices: (i) active wireless-powered devices (AWPDs) denoted by $\mathcal{A} \triangleq(1, \ldots, a, \ldots, A)$, (ii) passive wirelesspowered devices (PWPDs) denoted by $\mathcal{P} \triangleq(1, \ldots, p, \ldots, P)$, and (iii) hybrid wireless-powered devices (HWPDs) denoted by $\mathcal{H} \triangleq(1, \ldots, h, \ldots, H)$. Intuitively, an AWPD is equipped with energy harvesting and wireless transmission circuits. In this way, the AWPD can harvest RF energy when the RF

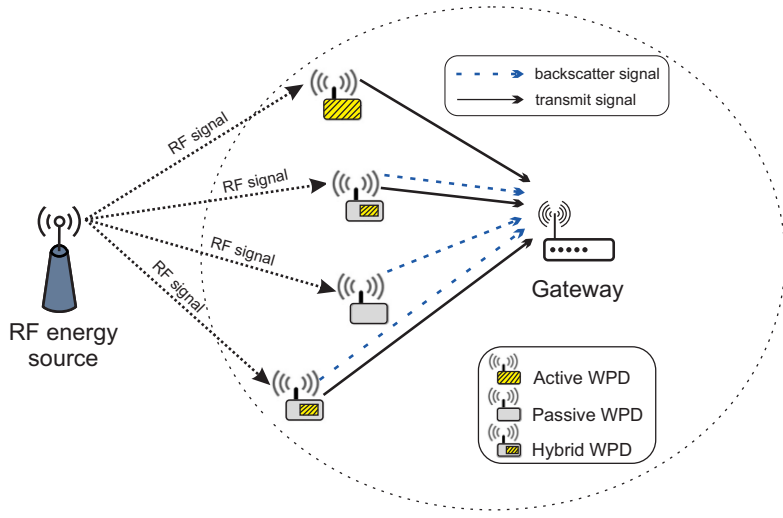

Fig. 1: System model for the considered HWPBN.

energy source transmits signals and use the harvested energy to actively transmit data to the gateway, i.e., harvest-thentransmit (HTT) mode. In contrast, a PWPD is a small device which is equipped with a backscatter circuit (without using battery, e.g., ATM and security cards) to backscatter signals to transmit information when the RF energy source transmits signals, i.e., backscatter mode. Finally, an HWPD is equipped with all components, i.e., energy harvesting, backscattering, and active transmission circuits, and it can choose to work in either the HTT or backscatter mode.

Fig. 2 shows a normalized time frame for the HWPBN which is divided into two successive periods, i.e., passive backscatter (PB) and active transmission (AT) periods to maximize energy efficiency for the HWPBN. In the PB period, the RF energy source transmits RF signals, i.e., energy transmission period, and thus all IoT devices equipped with energy harvesting circuits can harvest energy from the RF signals. In addition, the PWPDs and HWPDs can transmit their data by backscattering the RF signals of the RF energy source based on the backscattering circuits. In the AT period, the RF energy source is idle, i.e., sleeping period. Thus, only the AWPDs and HWPDs can actively transmit signals to the gateway if they have sufficient energy. As there are multiple IoT devices working in both periods, we adopt the TDMA mechanism to avoid collisions among transmissions due to its simplicity and efficiency [11], [12].

We denote the normalized time period of the RF energy source being idle by $\beta . \boldsymbol{\theta} \triangleq\left(\theta_{1}, \ldots, \theta_{p}, \ldots, \theta_{P}\right)^{\mathrm{T}}$ and $\boldsymbol{\nu} \triangleq\left(\nu_{1}, \ldots, \nu_{a}, \ldots, \nu_{A}\right)^{\mathrm{T}}$ denote the backscattering time of the PWPDs in the PB period and the transmission time of the AWPDs in the AT period, respectively. Similarly, the backscatter time and the transmission time of the HWPDs are represented by vectors $\boldsymbol{\tau} \triangleq\left(\tau_{1}, \ldots, \tau_{h}, \ldots, \tau_{H}\right)^{\mathrm{T}}$ and $\boldsymbol{\mu} \triangleq\left(\mu_{1}, \ldots, \mu_{h}, \ldots, \mu_{H}\right)^{\mathrm{T}}$, respectively. Note that the aforementioned variables must satisfy the following constraints:

$$
\left(\mathbf{C}_{1}\right) \quad\left\{\begin{array}{c}
\|\boldsymbol{\theta}\|+\|\boldsymbol{\tau}\|=\sum_{p=1}^{P} \theta_{p}+\sum_{h=1}^{H} \tau_{h} \leq(1-\beta), \\
\|\boldsymbol{\nu}\|+\|\boldsymbol{\mu}\|=\sum_{a=1}^{A} \nu_{a}+\sum_{h=1}^{H} \mu_{h} \leq \beta, \\
\theta_{p} \geq 0, \forall p \in \mathcal{P} \\
\nu_{a} \geq 0, \forall a \in \mathcal{A} \\
\tau_{h}, \mu_{h} \geq 0, \forall h \in \mathcal{H},
\end{array}\right.
$$




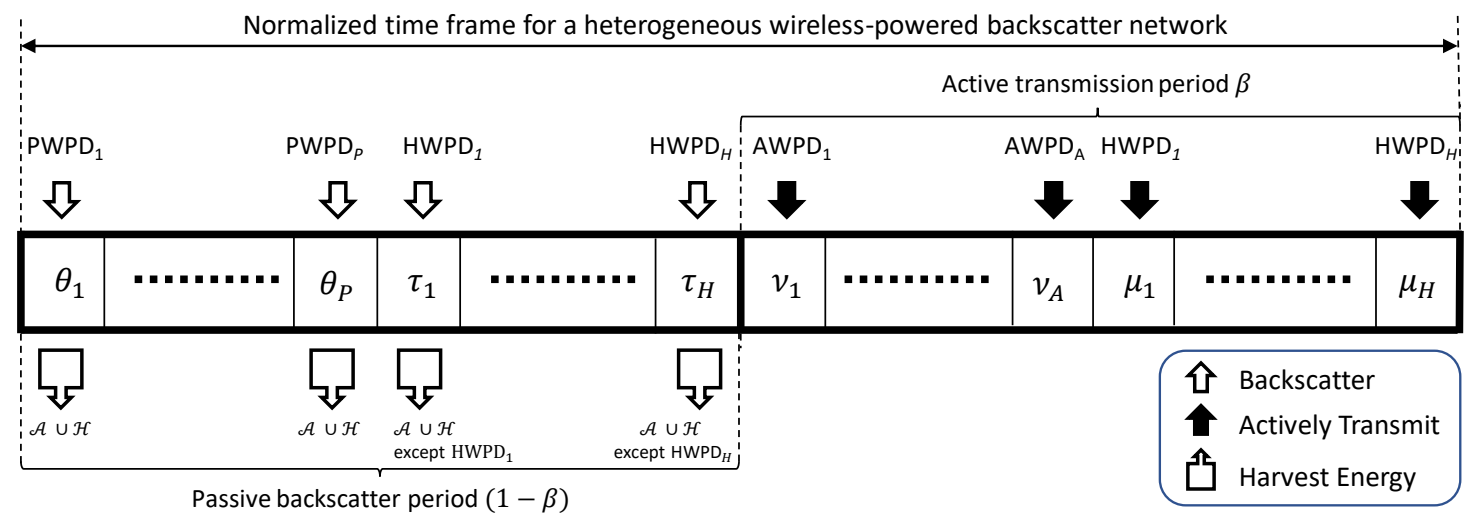

Fig. 2: Normalized time frame structure of the HWPBN.

where the first constraint ensures that the total backscatter time of all PWPDs and HWPDs will not exceed the energy transmission period, i.e., the PB period, and the second constraint guarantees that all AWPDs and HWPDs will only transmit data in the sleeping period of the RF energy source, i.e., the AT period.

To maximize the energy efficiency, the RF energy source can adjust its transmission power $P^{T}$ based on the network parameters such as the number of IoT devices in each group, backscatter rates, transmission efficiency and backscatter efficiency of IoT devices. In this paper, we jointly consider the energy management problem at the RF energy source and the time scheduling for IoT devices to maximize the network throughput.

\section{PRoblem Formulation}

The network throughput is defined as the total number of transmitted information bits by all IoT devices in the network over the normalized time frame. We define $R_{\text {sum }}$ as the network throughput which is defined as follows:

$R_{\text {sum }}=R^{b s}+R^{t r}=\sum_{p=1}^{P} R_{p}^{b s}+\sum_{h=1}^{H} R_{h}^{b s}+\sum_{a=1}^{A} R_{a}^{t r}+\sum_{h=1}^{H} R_{h}^{t r}$,

where $R^{b s}$ is the total throughput achieved by backscattering communications in the PB period, which is defined as the sum of all transmitted bits by the PWPDs and HWPDs, i.e., $R^{b s}=\sum_{p=1}^{P} R_{p}^{b s}+\sum_{h=1}^{H} R_{h}^{b s} . R^{t r}$ is the total throughput obtained by active transmissions in the AT period, which includes all transmitted bits of the AWPDs and HWPDs, i.e., $R^{t r}=\sum_{a=1}^{A} R_{a}^{t r}+\sum_{h=1}^{H} R_{h}^{t r}$.

\section{A. Passive Backscatter Period}

In the passive backscatter period, as the RF energy source broadcasts RF signals, the AWPDs can harvest energy from the RF signals and the PWPDs can transmit data by backscattering such RF signals. Furthermore, the HWPDs can select to work in either the backscatter mode or HTT mode.
1) Backscatter Process: Let $W_{p}$ and $W_{h}$ denote the achievable backscatter rates $^{1}$ of the PWPD- $p$ and the HWPD- $h$, respectively. Then, the total number of bits transmitted by the PWPDs and HWPDs in the PB period is expressed as follows:

$$
R^{b s}=\sum_{p=1}^{P} \eta_{p} W_{p} \theta_{p}+\sum_{h=1}^{H} \eta_{h} W_{h} \tau_{h}
$$

where $\eta_{p}$ and $\eta_{h}$ are the backscattering efficiency coefficients of the PWPD- $p$ and the HWPD- $h$, respectively. It is worth noting that the backscatter rate depends on values of resistors and capacitors in the RC circuit [13].

2) Energy Harvesting Process: Both the AWPDs and HWPDs can harvest energy from the RF energy source in the PB period. The harvested energy is then used to actively transmit data in the AT period. The amount of energy received by the AWPDs and HWPDs from the RF signals can be calculated by Friis equation [14] as follows:

$$
\left\{\begin{array}{c}
P_{a}^{R}=\delta_{a} P^{T} \frac{G^{T} G_{a}^{R} \lambda^{2}}{\left(4 \pi d_{a}\right)^{2}}, \forall a \in \mathcal{A} \\
P_{h}^{R}=\delta_{h} P^{T} \frac{G^{T} G_{h}^{R} \lambda^{2}}{\left(4 \pi d_{h}\right)^{2}}, \forall h \in \mathcal{H}
\end{array}\right.
$$

where $P^{T}$ and $G^{T}$ are the transmission power and the antenna gain of the RF energy source, respectively. $G_{a}^{R}$ and $G_{h}^{R}$ are the antenna gains of the AWPD- $a$ and HWPD- $h$, respectively. $\lambda$ is the wavelength of the RF signal, $d_{a}$ and $d_{h}$ are the distances between the RF energy source and the AWPD- $a$ and HWPD$h$, respectively. $\delta_{a}$ and $\delta_{h}$ are energy harvesting efficiency coefficients of the AWPD- $a$ and HWPD- $h$, respectively. Note that, when an HWPD backscatters RF signals to the gateway, other HWPDs still can harvest energy and store the harvested energy in their batteries. In the PB period, all AWPDs can harvest energy during this period, i.e., $(1-\beta)$, while each HWPD- $h$ can only harvest energy in a period of $\left(1-\beta-\tau_{h}\right)$. Thus, the total harvested energy of the AWPDs and HWPDs can be calculated as follows:

$$
\left\{\begin{array}{l}
E_{a}=(1-\beta) P_{a}^{R}, \forall a \in \mathcal{A}, \\
E_{h}=\left(1-\beta-\tau_{h}\right) P_{h}^{R}, \forall h \in \mathcal{H} .
\end{array}\right.
$$

\footnotetext{
${ }^{1}$ The number of bits decoded successfully at the receiver by using the bistatic backscatter communication.
} 


\section{B. Active Transmission Period}

There are only the AWPDs and HWPDs that can operate in the AT period to transmit their data by using their RF circuits. As mentioned in the previous section, each AWPD- $a$ is allocated a time slot $\nu_{a}$ and HWPD- $h$ is allocated a time slot $\mu_{h}$ to transmit data. We assume that, the AWPDs and HWPDs will utilize all their harvested energy in the PB period to actively transmit their data, the transmission power of the AWPD- $a$ and HWPD- $h$ in the AT period can be achieved by:

$$
\left\{\begin{array}{l}
P_{a}^{t}=\frac{E_{a}}{\nu_{a}}, \forall a \in \mathcal{A}, \\
P_{h}^{t}=\frac{E_{h}}{\mu_{h}}, \forall h \in \mathcal{H} .
\end{array}\right.
$$

Then, the transmission rates of these devices can be obtained by:

$$
\left\{\begin{array}{l}
r_{a}=\varphi_{a} \Lambda \log _{2}\left(1+\frac{g_{a} P_{a}^{t}}{N_{a}^{o}}\right), \forall a \in \mathcal{A}, \\
r_{h}=\varphi_{h} \Lambda \log _{2}\left(1+\frac{g_{h} P_{h}^{t}}{N_{h}^{0}}\right), \forall h \in \mathcal{H},
\end{array}\right.
$$

where $\varphi_{a}$ and $\varphi_{h} \in(0,1)$ are the transmission efficiency coefficients of the AWPD- $a$ and HWPD- $h$, respectively. $\Lambda$ is the bandwidth of the channel from the IoT devices to the gateway. $g_{a}$ and $g_{h}$ are the channel gains of the AWPD- $a$ and HWPD- $h$, respectively. $N_{a}^{0}$ and $N_{h}^{0}$ are the noise on the communication channel of the AWPD- $a$ and the HWPD- $h$, respectively. The total throughputs of the AWPDs and HWPDs in the AT period are then obtained by:

$$
\begin{aligned}
R^{t r}= & \sum_{a=1}^{A} R_{a}^{t r}+\sum_{h=1}^{H} R_{h}^{t r}=\sum_{a=1}^{A} \nu_{a} \kappa_{a} \log _{2}\left[1+\gamma_{a} \frac{(1-\beta) P_{a}^{R}}{\nu_{a}}\right] \\
& +\sum_{h=1}^{H} \mu_{h} \kappa_{h} \log _{2}\left[1+\gamma_{h} \frac{(1-\beta) P_{h}^{R}}{\mu_{h}}\right]
\end{aligned}
$$

where $\kappa_{a}=\varphi_{a} \Lambda, \kappa_{h}=\varphi_{h} \Lambda, \gamma_{a}=g_{a} / N_{a}^{0}, \gamma_{h}=g_{h} / N_{h}^{0}$.

After that, the achieved network throughput of the HWPBN system can be determined as shown in (9), where $\phi_{a}=$ $\gamma_{a} \delta_{a} \frac{G^{T} G_{a}^{R} \lambda^{2}}{\left(4 \pi d_{a}\right)^{2}}$ and $\phi_{h}=\gamma_{h} \delta_{h} \frac{G^{T} G_{h}^{R} \lambda^{2}}{\left(4 \pi d_{h}\right)^{2}}$.

Due to energy limitation of the RF energy source, its energy consumption must be controlled under a threshold $E_{S}^{0}$ as follows:

$$
\left(\mathbf{C}_{2}\right) \quad P^{T}(1-\beta) \leq E_{S}^{0} .
$$

Moreover, the transmission power of the RF energy source must follow the standard FCC for RF devices [15] defined by:

$$
\text { (C) } \frac{E_{S}^{0}}{1-\beta} \leq P T^{*} .
$$

Similarly, the transmission power of the AWDPs and HWDPs in the AT period also must be under thresholds $P_{a}^{0}$ and $P_{h}^{0}$, respectively, as follows:

$$
\left(\mathbf{C}_{4}\right) \quad\left\{\begin{array}{l}
\frac{P_{a}^{R}(1-\beta)}{\nu_{a}} \leq P_{a}^{0}, \forall a \in \mathcal{A}, \\
\frac{P_{h}^{\nu^{2}}\left(1-\beta-\tau_{h}\right)}{\mu_{h}} \leq P_{h}^{0}, \forall h \in \mathcal{H} .
\end{array}\right.
$$

In addition, the total harvested energy of the AWPDs in the time period $(1-\beta)$ and the HWPDs in the time period $(1-$ $\left.\beta-\tau_{h}\right)$ must be ensured to be sufficient for their operation requirements, i.e., $E_{a}^{0}$ and $E_{h}^{0}$, respectively:

$$
\left(\mathbf{C}_{5}\right) \quad\left\{\begin{array}{l}
P_{a}^{R}(1-\beta) \geq E_{a}^{0}, \forall a \in \mathcal{A}, \\
P_{h}^{R}\left(1-\beta-\tau_{h}\right) \geq E_{h}^{0}, \forall h \in \mathcal{H} .
\end{array}\right.
$$

Finally, the joint optimization problem can be formulated as follows:

$$
\begin{array}{cc}
\left(\mathbf{P}_{1}\right) & \max _{\left(\boldsymbol{\theta}, \boldsymbol{\nu}, \boldsymbol{\tau}, \boldsymbol{\mu}, P^{T}\right)} R_{\text {sum }}, \\
\text { s.t } & \left(\mathbf{C}_{1}\right),\left(\mathbf{C}_{2}\right),\left(\mathbf{C}_{3}\right),\left(\mathbf{C}_{4}\right), \text { and }\left(\mathbf{C}_{5}\right) .
\end{array}
$$

\section{JOINT OPTIMAL TRANSMISSION POWER AND TIME RESOURCE ALLOCATION}

The objective function $R_{\text {sum }}$ w.r.t. $\left(\boldsymbol{\theta}, \boldsymbol{\mu}, \boldsymbol{\tau}, \boldsymbol{\nu}, P^{T}\right)$ in $\left(\mathbf{P}_{1}\right)$ expressed in (14) is a non-convex function due to the strong relation among variables, i.e., the communication time of the HWPD- $h\left(\tau_{h}, \mu_{h}\right)$ and the received power at the HWPD- $h$ $P_{h}^{R}$ as shown in (8). Hence, the optimal solution is intractable to obtain directly. Thus, we first prove that $\left(\mathbf{P}_{1}\right)$ can be transformed to an equivalent convex problem as shown below:

$$
\begin{array}{cc}
\left(\mathbf{P}_{2}\right) & \max _{(\boldsymbol{\theta}, \boldsymbol{\nu}, \boldsymbol{\tau}, \boldsymbol{\mu})} \hat{R}_{\text {sum }}, \\
\text { s.t } & \left(\mathbf{C}_{1}\right),\left(\mathbf{C}_{3}\right),\left(\mathbf{C}_{4}\right), \text { and }\left(\mathbf{C}_{5}\right) .
\end{array}
$$

Theorem 1. The optimization problem $\left(\mathbf{P}_{1}\right)$ with constraints $\left(\mathbf{C}_{1}\right)-\left(\mathbf{C}_{5}\right)$ is equivalent to the optimization problem $\left(\mathbf{P}_{2}\right)$ with constraints $\left(\mathbf{C}_{1}\right),\left(\mathbf{C}_{3}\right),\left(\mathbf{C}_{4}\right)$, and $\left(\mathbf{C}_{5}\right)$.

Proof. Due to the limited space, we briefly explain the proof of Theorem 2 as follows. Given an optimal set of $\left(\boldsymbol{\theta}^{*}, \boldsymbol{\nu}^{*}, \boldsymbol{\tau}^{*}, \boldsymbol{\mu}^{*}\right)$, the objective function $R_{\text {sum }}$ becomes a logarithm function of $P^{T}$ which is a monotonically increasing function as shown in (16). Therefore, the objective function $\tilde{R}_{\text {sum }}\left(P^{T}\right)$ has the maximum value at the maximum value of $P^{T}$. From the energy constraint $\left(\mathbf{C}_{2}\right)$ shown in (10), we have the maximum value of transmission power of the $\mathrm{RF}$ energy source at $E_{S}^{0} /(1-\beta)$ due to $(1-\beta)>0$. Thus, with the maximum value of $P^{T}$, the objective function can be transformed to an equivalent function $\hat{R}_{\text {sum }}$ w.r.t. $(\boldsymbol{\theta}, \boldsymbol{\mu}, \boldsymbol{\tau}, \boldsymbol{\nu})$ which is defined in (17). As a result, we can find the optimal solution of the primal optimization problem $\left(\mathbf{P}_{1}\right)$ by solving the equivalent optimization problem $\left(\mathbf{P}_{2}\right)$.

To find the optimal solution for the optimization problem $\left(\mathbf{P}_{2}\right)$, we first prove that the objective function $\hat{R}_{\text {sum }}$ is a concave function as shown in Theorem 2.

Theorem 2. The objective function $\hat{R}_{\text {sum }}$ is a concave function w.r.t. $(\boldsymbol{\theta}, \boldsymbol{\nu}, \boldsymbol{\tau}, \boldsymbol{\mu})$ satisfying the constraint $\left(\mathbf{C}_{1}\right)$.

Proof. In the objective function $\hat{R}_{\text {sum }}$ in (17), the first term is a linear function of $\theta_{p}$. Therefore, we only need to prove that the last two terms are concave functions. We first denote three terms in the objective function $\hat{R}_{\text {sum }}$ in (17) by $F_{p}(\boldsymbol{\theta})=\sum_{p=1}^{P} f_{p}\left(\theta_{p}\right), F_{a}(\boldsymbol{\nu})=\sum_{a=1}^{A} f_{a}\left(\nu_{a}\right)$, and $F_{h}(\boldsymbol{\tau}, \boldsymbol{\mu})=\sum_{h=1}^{H} f_{h}\left(\tau_{h}, \mu_{h}\right)$, where

$$
\begin{cases}f_{p}\left(\theta_{p}\right)= & \eta_{p} W_{p} \theta_{p}, \\ f_{a}\left(\nu_{a}\right)= & \nu_{a} \kappa_{a} \log _{2}\left(1+\frac{\phi_{a} E_{S}^{0}}{\nu_{a}}\right), \\ f_{h}\left(\tau_{h}, \mu_{h}\right)= & \eta_{h} W_{h} \tau_{h} \\ & +\mu_{h} \kappa_{h} \log _{2}\left[1+\phi_{h} \frac{\left(1-\beta-\tau_{h}\right) E_{S}^{0}}{(1-\beta) \mu_{h}}\right] .\end{cases}
$$

It is worth noting that in the optimization problem $\left(\mathbf{P}_{\mathbf{2}}\right)$, given a value of $P^{T}$, we have $(1-\beta)=E_{S}^{0} / P^{T}$ is a constant 


$$
\begin{aligned}
& R_{\text {sum }}\left(\boldsymbol{\theta}, \boldsymbol{\mu}, \boldsymbol{\tau}, \boldsymbol{\nu}, P^{T}\right) \\
&= \sum_{p=1}^{P} \eta_{p} \mathrm{~W}_{p} \theta_{p}+\sum_{a=1}^{A} \nu_{a} \kappa_{a} \log _{2}\left[1+\gamma_{a} \frac{(1-\beta) P_{a}^{R}}{\nu_{a}}\right]+\sum_{h=1}^{H} \eta_{h} \mathrm{~W}_{h} \tau_{h}+\sum_{h=1}^{H} \mu_{h} \kappa_{h} \log _{2}\left[1+\gamma_{h} \frac{\left(1-\beta-\tau_{h}\right) P_{h}^{R}}{\mu_{h}}\right] \\
&= \sum_{p=1}^{P} \eta_{p} \mathrm{~W}_{p} \theta_{p}+\sum_{a=1}^{A} \nu_{a} \kappa_{a} \log _{2}\left[1+\phi_{a} \frac{(1-\beta) P^{T}}{\nu_{a}}\right]+\sum_{h=1}^{H}\left\{\eta_{h} \mathrm{~W}_{h} \tau_{h}+\mu_{h} \kappa_{h} \log _{2}\left[1+\phi_{h} \frac{\left(1-\beta-\tau_{h}\right) P^{T}}{\mu_{h}}\right]\right\} . \\
& \tilde{R}_{\text {sum }}\left(P^{T}\right)=\sum_{p=1}^{P} \eta_{p} \mathrm{~W}_{p} \theta_{p}^{*}+\sum_{a=1}^{A} \nu_{a}^{*} \kappa_{a} \log _{2}\left[1+\phi_{a} \frac{(1-\beta) P^{T}}{\nu_{a}^{*}}\right]+\sum_{h=1}^{H}\left\{\eta_{h} \mathrm{~W}_{\mathrm{h}} \tau_{h}^{*}+\mu_{h}^{*} \kappa_{h} \log _{2}\left[1+\phi_{h} \frac{\left(1-\beta-\tau_{h}^{*}\right) P^{T}}{\mu_{h}^{*}}\right]\right\} . \\
& \hat{R}_{\text {sum }}(\boldsymbol{\theta}, \boldsymbol{\mu}, \boldsymbol{\tau}, \boldsymbol{\nu})=\sum_{p=1}^{P} \eta_{p} \mathrm{~W}_{p} \theta_{p}+\sum_{a=1}^{A} \nu_{a} \kappa_{a} \log _{2}\left[1+\frac{\phi_{a} E_{S}^{0}}{\nu_{a}}\right]+\sum_{h=1}^{H}\left\{\eta_{h} \mathrm{~W}_{h} \tau_{h}+\mu_{h} \kappa_{h} \log _{2}\left[1+\phi_{h} \frac{\left(1-\beta-\tau_{h}\right) E_{S}^{0}}{(1-\beta) \mu_{h}}\right]\right\} .
\end{aligned}
$$

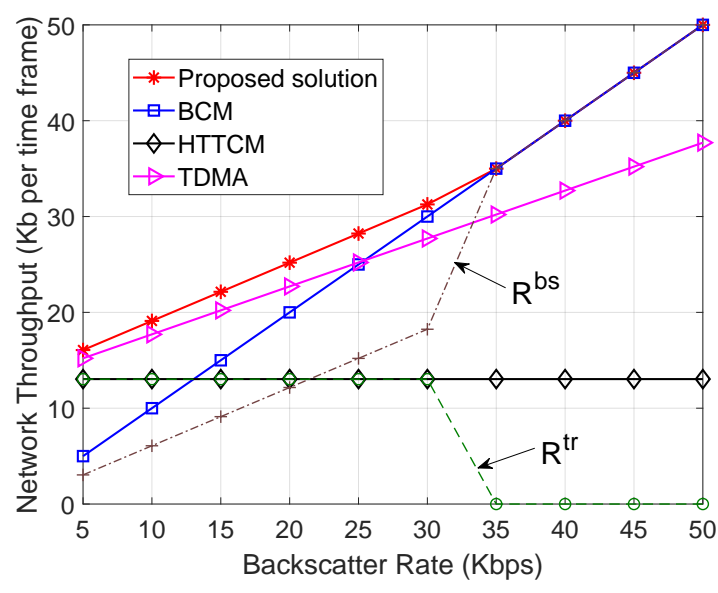

Fig. 3: Network throughput vs backscatter rate.

value. Thus, the function $f_{h}$ is only with respect to variables $\left(\tau_{h}, \mu_{h}\right)$. Then, we have the following lemmas:

Lemma 1. The sub-function $F_{a}(\boldsymbol{\nu})$ is a concave function w.r.t. $\nu_{a}(\forall a \in \mathcal{A})$ satisfying the constraint $\left(\mathbf{C}_{1}\right)$.

Lemma 2. The sub-function $F_{h}(\boldsymbol{\tau}, \boldsymbol{\mu})$ is a concave function w.r.t. $\left(\tau_{h}, \mu_{h}\right)(\forall h \in \mathcal{H})$ satisfying the constraint $\left(\mathbf{C}_{\mathbf{1}}\right)$.

The proofs of Lemma 1 and Lemma 2 are presented in Appendix A and Appendix B, respectively.

According to the Lemma 1 and Lemma 2, the objective function $\hat{R}_{\text {sum }}$ is a concave function w.r.t. $(\boldsymbol{\theta}, \nu, \tau, \boldsymbol{\mu})$.

There are some convex optimization tools which can be adopted to find the optimal solution of the optimization problem $\left(\mathbf{P}_{2}\right)$ due to its concavity. In this paper, we utilize the interior-point method which is an efficient solution to solve convex optimization problems with inequality constraints.

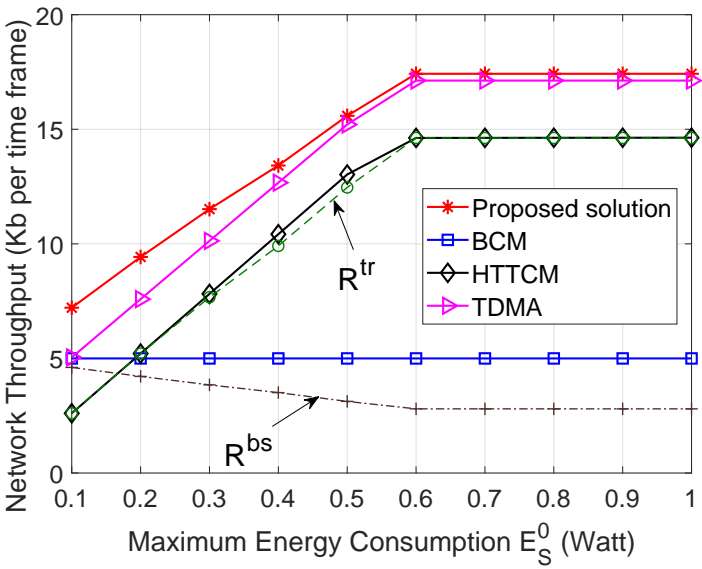

Fig. 4: Network throughput vs energy consumption threshold of RF source.

\section{NUMERICAL RESUlTS}

We perform the simulations using MATLAB to evaluate the performance of the proposed solution under different parameter settings. Followed by the FCC Rules for unlicensed wireless equipment operating in the ISM bands [15] (afar.net/tutorials/fcc-rules/), we set the frequency of RF carrier signals and the antenna gain of the dedicated RF energy source at $2.4 \mathrm{GHz}$ and $6 \mathrm{dBi}$, respectively. Under this setting, the transmission power of the RF energy source must be controlled under the threshold of $30 \mathrm{dBm}$ (i.e., 1 Watt). The bandwidth of the RF carrier signals is set at $10 \mathrm{MHz}$ and the antenna gains of the AWPDs/HWPDs are also set at 6 $\mathrm{dBi}$ [16]. The distances between the RF source and the IoT devices are 20 meters. The maximum energy consumption threshold of the RF source $E_{S}^{0}$ in the normalized time frame is set at $0.5 \mathrm{~W}$. The energy harvesting efficiency and the data transmission efficiency of both the AWPDs and HWPDs are $\varphi=0.6$ and $\delta=0.5$, respectively. Unless otherwise stated, the 


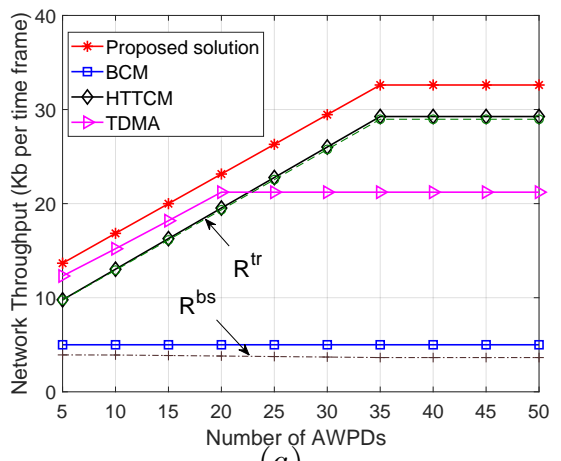

(a)

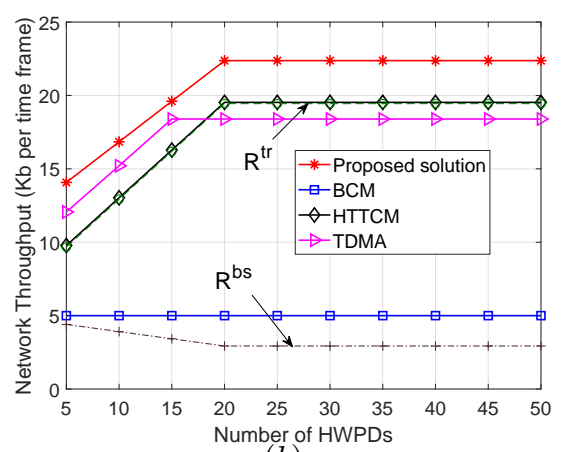

$(b)$

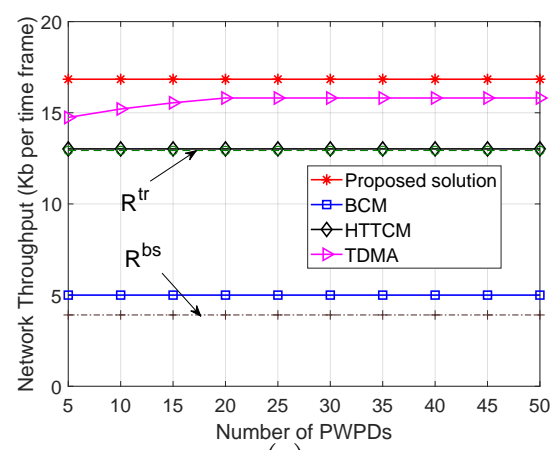

(c)

Fig. 5: Network throughput vs (a) number of AWPDs, (b) number of HWPDs, and (c) number of PWPDs.

backscatter rate for the PWPDs and HWPDs is $5 \mathrm{kpbs}$, and the number of IoT devices is 10 for each type. In the following, we evaluate the performance of the proposed solution by comparing with three other conventional methods, i.e., using the HTT communication method only [8] (HTTCM), backscattering communication method (BCM) only [13], and TDMA mechanism. For the TDMA mechanism, time resources are allocated equally for all IoT device types.

\section{A. Network Performance under a Fixed Number of IoT De- vices}

To evaluate the performance of the proposed solution, we first consider the scenario with a fixed number of IoT devices, i.e., $A=P=H=10$, under different backscatter rates and energy consumption thresholds of the RF energy source. First, Fig. 3 shows the network throughput as the backscatter rate increases from $5 \mathrm{kbps}$ to $50 \mathrm{kbps}$. The energy consumption threshold $E_{S}^{0}$, and the maximum transmission power $P T^{*}$ of the RF source are set at 0.5 and 0.9 Watt, respectively, (to follow the FFC rules [15]). As observed in Fig. 3, the network throughputs obtained by the proposed solution, BCM and TDMA mechanism increase as the backscatter rate increases. For the proposed solution, when the backscatter rate is low (less than $20 \mathrm{kbps}$ ), IoT devices will be scheduled to spend more time for harvesting energy and transmitting data, and thus $R^{t r}$ is higher than $R^{b s}$ as shown in Fig. 3. However, when the backscatter rate is increased, the proposed solution will prefer backscattering communications, and when the backscatter rate is higher than $35 \mathrm{kbps}$, all devices are scheduled to use backscatter communications to achieve the best network throughput. This result also reveals an important information in managing heterogeneous devices in HWPBNs. In particular, when the backscatter rate is high, we should impose constraints to the AWPDs to guarantee their operations. In contrast, the scheduled backscatter time and transmission time for all IoT devices in the TDMA mechannism are equal, and thus the network throughput of the TMDA solution increases linearly according to the backscatter rate.

We then fix the backscatter rate at $5 \mathrm{kbps}$ and increase the energy consumption threshold of the RF energy source from 0.1 to 1 Watt to evaluate the network performance. As shown in Fig. 4, as the maximum energy consumption threshold increases, the amount of harvested energy will be increased, and thus network throughputs obtained by solutions using HTT mode will be enhanced. However, interestingly, when the maximum energy threshold is higher than 0.6 , the network throughputs obtained by all solutions remain unchanged. The reason is that due to the low-power consumption requirements, IoT transmission powers are often set at low levels (we set the transmission power of IoT devices at $5 \times 10^{-5}$ Watt in this case), and thus given a dedicated transmission time, IoT devices cannot use all harvested energy amount for their transmission. Consequently, the network throughput cannot be enhanced. This information is also very important when we control transmission power of the RF energy source to maximize energy efficiency for HWPBNs.

\section{B. Network Performance under Different Numbers of IoT Devices}

We now evaluate performance of the system by varying the number of IoT devices of one type between 5 and 50 , and fixing the number of IoT devices of other types at 10 . Other parameters are set to be the same for all three cases, i.e., $5 \mathrm{kbps}$ for backscatter rate, 0.5 and 0.9 Watts for the maximum energy consumption threshold and the maximum transmission power of the RF source, respectively. In general, the proposed solution always achieves the highest performance compared with other solutions in all cases as shown in Fig. 5. In particular, in Fig. 5(a), as the number of AWPDs increases, the network throughputs obtained by the proposed solution, HTTCM and TDMA mechanism will be increased. This is because multiple IoT devices can harvest energy simultaneously, and thus the energy efficiency from RF energy source can be maximized. However, when the number of AWPDs is too many, i.e., higher than 35 devices for the proposed solution and HTTCM, and 20 devices for the TDMA mechanism, respectively, there is no more gain for the network throughputs due to the limitations of transmission time and transmission power of IoT devices. Similar trends can be obtained in Fig. 5(b) when we increase the number of HWPDs. Note that, the network throughput of the proposed solution is not influenced by the number of PWPDs as shown in Fig. 5(c) because in this case the backscatter rate is set at low level, i.e., $5 \mathrm{kbps}$, and thus the proposed solution will prefer the HTT mode. For the 
above reason, moreover, the network throughput of the TDMA mechanism is only affected by the number of PWPDs when the number of IoT devices is small, i.e., less than 25 devices.

\section{CONCLUSION}

In this paper, we have proposed the novel approach to jointly address energy and communication problems for heterogeneous IoT low-power wireless communication networks. In particular, we have considered a heterogeneous wirelesspowered backscatter network with three different IoT device types under diverse hardware configurations and dissimilar communication and energy requirements. To simultaneously meet the IoT devices' requirements and achieve the best performance for the network, we have proposed the solution to not only tradeoff operation time for IoT devices, e.g., backscatter, energy harvesting, and active transmission time, but also minimize energy consumption for the RF energy source. The simulation results then show that our proposed solution always achieves the best performance compared with other conventional methods. In addition, the simulation results also reveal some important information in controlling energy supply and managing low-power IoT devices in heterogeneous wireless communication networks.

\section{APPENDIX A}

\section{THE PROOF OF LEMMA 1}

To prove $F_{a}(\boldsymbol{\nu})$ is a concave function, we first prove that the function $f_{a}\left(\nu_{a}\right)$ is concave. Specifically, we can derive the second partial derivative of $f_{a}$ w.r.t. $\nu_{a}$ as follows:

$$
\nabla^{2} f_{a}\left(\nu_{a}\right)=\frac{\partial^{2} f_{a}}{\partial^{2} \nu_{a}}=-\frac{\kappa_{a}\left(\phi_{a} E_{S}^{0}\right)^{2}}{\nu_{a}\left(\nu_{a}+\phi_{a} E_{S}^{0}\right)^{2} \ln 2} .
$$

It can be seen that the second derivative of $f_{a}$ w.r.t. $\nu_{a}$ less than or equal to zero because $\kappa_{a} \geq 0, \nu_{a}>0$, and $\phi_{a}>$ $0, \forall a \in \mathcal{A}$. In the case $\nu_{a}=0$, then the function $f_{a}\left(\nu_{a}\right)=0$. Hence, the function $f_{a}\left(\nu_{a}\right)$ is concave $(\forall a \in \mathcal{A})$, then the sub-function $F_{a}(\boldsymbol{\nu})=\sum_{a=1}^{A} f_{a}\left(\nu_{a}\right)$ is also concave.

\section{APPENDIX B}

\section{THE PROOF OF LEMMA 2}

Due to the limited space, we briefly explain the proof of Lemma 2 as below.

Similar to the proof of the Lemma 1, we first find the second derivative of the function $f_{h}$ w.r.t. $\left(\tau_{h}, \mu_{h}\right)$ which is well known as the Hessian matrix $\mathbf{L}_{h}$ obtained in follows:

$$
\mathbf{L}_{h}=\left[\begin{array}{cc}
\frac{\partial^{2} f_{h}}{\partial^{2} \tau_{h}} & \frac{\partial^{2} f_{h}}{\partial \tau_{h} \partial \mu_{h}} \\
\frac{\partial^{2} f_{h}}{\partial \mu_{h} \partial \tau_{h}} & \frac{\partial^{2} f_{h}}{\partial^{2} \mu_{h}}
\end{array}\right]
$$

Then, we prove that $\mathbf{L}_{h}$ is a negative semi-definite matrix $(\forall h \in \mathcal{H})$. Given an arbitrary real vector $\boldsymbol{u}=\left[u_{1}, u_{2}\right]$, we have:

$$
\mathbf{u}^{\mathrm{T}} \mathbf{L}_{h} \mathbf{u}=-\frac{\kappa_{h}}{\mu_{h}\left(1+z_{h}\right)^{2} \ln 2}\left[\frac{\phi_{h} E_{S}^{0}}{(1-\beta)} u_{1}+z_{h} u_{2}\right]^{2},
$$

where $z_{h}=\phi_{h} \frac{\left(1-\beta-\tau_{h}\right) E_{S}^{0}}{(1-\beta) \mu_{h}}$. For $\kappa_{h} \geq 0, \mu_{h}>0,(\forall h \in \mathcal{H})$, we have $\mathbf{u}^{\mathrm{T}} \mathbf{L}_{h} \mathbf{u} \leq 0,(\forall h \in \mathcal{H})$. This means that $\mathbf{L}_{h}$ is a negative semi-definite matrix $(\forall h \in \mathcal{H})$. Especially, when $\mu_{h}=0$, the function $f_{h}\left(\tau_{h}, \mu_{h}\right)=0$. Hence, the function $f_{h}\left(\tau_{h}, \mu_{h}\right)$ is a concave function $(\forall h \in \mathcal{H})$. Because $F_{h}(\boldsymbol{\tau}, \boldsymbol{\mu})=\sum_{h=1}^{H} f_{h}\left(\tau_{h}, \mu_{h}\right)$, we then derive that $F_{h}(\boldsymbol{\tau}, \boldsymbol{\mu})$ is also a concave function.

\section{REFERENCES}

[1] D. Evans, "The Internet of Things: How the Next Evolution of the Internet Is Changing Everything", Cisco Report White paper, 2011.

[2] N. V. Huynh and D. T. Hoang and X. Lu and D. Niyato and P. Wang and D. I. Kim, "Ambient backscatter communications: A contemporary survey," IEEE Communications Surveys Tutorials, 2018.

[3] S. N. Daskalakis, J. Kimionis, A. Collado, G. Goussetis, M. M. Tentzeris, and A. Georgiadis, "Ambient backscatterers using FM broadcasting for low cost and low power wireless applications," IEEE Transactions on Microwave Theory and Techniques, vol. 65, no. 12, pp. 5251-5262, Nov. 2017.

[4] P. Zhang, M. Rostami, P. Hu, and D. Ganesan, "Enabling practical backscatter communication for on-body sensors," in Proc. of the ACM SIGCOMM, pp. 370-383, Florianopolis, Brazil, Aug. 2016.

[5] A. N. Parks, A. Liu, S. Gollakota, and J. R. Smith, "Turbocharging ambient backscatter communication," in Proc. of the ACM SIGCOMM, pp. 619-630, Chicago, Illinois, USA, Aug. 2014.

[6] J. Kimionis, A. Bletsas, and J. N. Sahalos, "Bistatic backscatter radio for power-limited sensor networks," in Proc. of IEEE Global Communications Conference, pp. 353-358, Atlanta, GA, USA, Dec. 2013.

[7] J. Kimionis, A. Bletsas, and J. N. Sahalos, "Increased range bistatic scatter radio," IEEE Transactions on Communications, vol. 62, no. 3 , pp. 1091-1104, Mar. 2014.

[8] H. Ju and R. Zhang, "Throughput maximization in wireless powered communication networks," IEEE Transactions on Wireless Communications, vol. 13, no. 1, pp. 418-428, Jan. 2014.

[9] D. T. Hoang, D. Niyato, P. Wang, D. I. Kim, and Z. Han, "Ambient backscatter: A new approach to improve network performance for RFpowered cognitive radio networks," IEEE Transactions on Communications, vol. 65, no. 9, pp. 3659-3674, Sept. 2017.

[10] B. Lyu, Z. Yang, G. Gui, and Y. Feng, "Wireless powered communication networks assisted by backscatter communication," IEEE Access, vol. 5, pp. 7254-7262, Mar. 2017.

[11] X. Kang, C. K. Ho, and S. Sun,"Full-duplex wireless-powered communication network with energy causality," IEEE Transactions on Wireless Communications, vol. 14, no. 10, pp. 5539-5551, Oct. 2015.

[12] K. Chi, Y. Zhu, Y. Li, L. Huang, and M. Xia, "Minimization of transmission completion time in wireless powered communication networks," IEEE Internet of Things Journal, vol. 4, no. 5, pp. 16711683, Oct. 2017

[13] V. Liu, A. Parks, V. Talla, S. Gollakota, D. Wetherall, and J. R. Smith, "Ambient backscatter: Wireless communication out of thin air," in Proc. of the ACM SIGCOMM, pp. 39-50, Hong Kong, China, Aug. 2013.

[14] C. A. Balanis, Antenna Theory: Analysis and Design. New York, NY: Wiley, 2012.

[15] FCC Rules for RF devices, part 15, Oct 2018.

[16] D. Y. Kim and D. I. Kim, "Reverse-link interrogation range of a UHF MIMO-RFID system in Nakagami-m fading channels," IEEE Transactions on Industrial Electronics, vol. 57, no. 4, pp. 1468-1477, Apr. 2010. 